\title{
Experiencia intersubjetiva y reconocimiento de múltiples estados del self: cómo poder experimentar ser sujeto ${ }^{1}$
}

\author{
Silvia Jiménez Torres ${ }^{2}$ \\ IPSA-Levante, Valencia
}

\begin{abstract}
Este trabajo revisa el caso presentado por Federici. Livia necesita una base de apego seguro en la relación con su terapeuta. El diálogo natural y espontáneo entre Livia y Susanna favoreció el reconocimiento de múltiples estados del self. El diálogo permitió que se escucharan todas las voces, que se expresaran todos los diversos sí mismos. Tal diálogo fomenta el reconocimiento mutuo en la experiencia intersubjetiva y es la conexión emocional entre las personas que dialogan, directriz fundamental que les guía en este diálogo. Paciente y terapeuta conviven íntimamente el uno con el otro en el diálogo analítico e intentan el reconocimiento recíproco de la subjetividad del otro, logrando también un mayor desarrollo y reconocimiento de la propia subjetividad, a la vez que amplían sus horizontes de experiencia. La relación analítica con Susanna, permitió a Livia sentir que era alguien que podía distinguirse de los demás, le permite experimentar ser sujeto.
\end{abstract}

Palabras clave: Multiplicidad del Self, Intersubjetividad.

This paper reviews the case presented by Federici. Livia needs a base of secure attachment in the relationship with her therapist. The natural and spontaneous dialogue between Livia and Susanna allows the recognition of multiple self-states. Their dialogue allowed all voices that express all the various themselves be heard. Such dialogue fosters mutual recognition in the intersubjective experience, as the emotional connection between people that dialogue, a fundamental guideline to guide them in this dialogue. Patient and therapist intimately coexist with each other in the analytic dialogue and seek mutual recognition of the subjectivity of the other, also achieving greater development and recognition of the subjectivity, while expanding their horizons of experience. Within the analytic relationship with Susanna, Livia allowed to feel that it was someone who could be distinguished from others, it allows her to experience being subject.

Key Words: Intersubjectivity, Self Multiplicity

English Title: Intersubjective Experience and recognition of Self Multiplicity: How to experience to be a subject.

\section{Cita bibliográfica / Reference citation:}

Jiménez Torres, S. (2016). Experiencia intersubjetiva y reconocimiento de múltiples estados del self: cómo poder experimentar ser sujeto. Clínica e Investigación Relacional, 10 (1): 99-105. [ISSN 1988-

2939] [Recuperado de www.ceir.info ] DOI: 10.21110/19882939.2016.100107

\footnotetext{
1 Presentado en la 6a Reunión anual de IARPP-España, Valencia. 24 de Octubre 2015.

${ }^{2}$ Dra. en Psicología - Directora de formación de IPSA-Levante.
} 
En primer lugar agradecer a Joan Coderch, Susanna Federici y Malcom Slavin su presencia en estas Jornadas, así como acceder a exponer uno de sus casos en ellas, compartiendo con nosotros su experiencia y conocimientos.

Por otro lado, me gustaría agradecer a todos los asistentes vuestra presencia, a conferenciantes, a profesionales, a estudiantes, etc. Se que no es sencillo hacer un hueco en las agendas, máxime cuando la asistencia a estas Jornadas nos llega desde tantos países diferentes. Agradeceros hacer posible un espacio de encuentro y de intercambio de experiencias y conocimientos.

Cuando se propuso que fuera una de las discutidoras de Susana Federici, tuve un sentimiento inmediato de alegría y emoción. Compartir la discusión de un caso de Susanna Federici y con Malcom Slavin era algo que no había previsto. Por ello, cuando llegó a mis manos y comencé a leerlo, vino a mi cabeza la célebre frase de Bion "Sin memoria ni deseo" y los consejos de uno de mis maestros, Luís Cencillo, que nos decía "...es importantísimo mantener la atención abierta y desprejuiciada a todo lo que el discurso del paciente exprese".

Así que comencé a leer el caso que presentaría Susanna en estas Jornadas exactamente de esa forma. Además, hacía poco, había leído el primer libro en castellano de Sandra Buechler "Marcando la diferencia en las vidas de los pacientes" e, influenciada por él, pensé en observar en un primer momento las emociones que sentía con su lectura.

Leer el caso de Livia me hizo sentir, en primer lugar, ternura, tristeza, miedo, enfado y también, lucha y resistencia. Hacia y por Livia, hacia y por Susanna y también por qué no, hacía y por mí misma. Susanna siente, y mucho, con esta paciente. Evoca, en el trabajo con Livia, su propia experiencia. Su propia experiencia de sentir la necesidad de "no molestar", de ser una niña sensible que pasaba desapercibida ante su madre a diferencia de su hermano pequeño. Esto también evocó en mí la propia experiencia de haber sido una niña excesivamente buena y vinieron a mi mente las palabras "si naces más buena naces tonta" repetidas a menudo durante mi infancia. Una niña que "no hacía ruido", que intentaba pasar desapercibida y que, al igual que Livia y Susanna, sentía la necesidad de separarme del contexto en situaciones tensas. De hecho, lo hice. Y al leer a Susanna, y sentir a Livia, puedo volver a sentir, a palpar, a recordar, mi experiencia. $Y$ evoca en mí recuerdos de todos aquellos pacientes que estuvieron o están en una situación similar.

Así, inmediatamente comprendí el interés de Susanna en encontrarse con la niña solitaria, asustada y enfadada que se escondía en Livia, y no solamente con la mujer joven, competente y sensible. 
Siguiendo a Steven Stern (2002), los investigadores del desarrollo temprano ven la infancia como una serie de momentos intersubjetivos o secuencias de interacción. El niño, en cada uno de estos momentos intersubjetivos, trae una experiencia subjetiva primaria a la que da repuesta el cuidador. El estado interno del que partía el niño es transformado por la interacción que se produce en el transcurso de cada secuencia interactiva; y, el niño internaliza las representaciones de esa secuencia de interacción transformacional mediante numerosas repeticiones de momentos similares. Se considera que la base de la estructura psicológica está formada por estas representaciones pre-simbólicas internalizadas (Stern, 1985; Beebe, Lachmann y Jaffe, 1997; Beebe y Lachmann, 1998) y se piensa que es este tipo de estructura la que provee la base para la multiplicidad de la experiencia del self. "La pequeña novia de papá", terrorífica frase, que resume a la perfección el dolor, la angustia que debía sentir Livia al tener que compartir los estados afectivos de su padre, estados afectivos que no le pertenecían, y que le "aniquilaban". Pero a la vez, le hacían sentir que ella tenía un lugar especial con su padre. Así, poco a poco, Livia pudo aprender a aniquilar su propia subjetividad y a enfocarse totalmente en el otro, algo paralelo al concepto de acomodación patológica de Brandchaft. Susanna expone cómo además de los modos despóticos y obsesivos del padre de Livia, su madre era igual. De niña, Livia, no tuvo a nadie que pudiera expresarle en palabras que estaba experimentando una angustia, una desesperación que realmente no le pertenecían, sino que pertenecían a su padre. Y ocurrió, que ese tipo de experiencias quedaron "no formuladas" como diría Donnel Stern. Y, a partir de repetidas experiencias de este tipo, Livia aprendió a ser buena, a no llorar para no preocupar a papa, etc. Creo interesante apuntar aquí, dos fenómenos que Paul Wachtel (2008) considera tienden a ocurrir en el curso del desarrollo. En primer lugar, una expansión y consolidación de los patrones auto-perpetuados cíclicos que caracterizan la vida de la persona, los cuales se arraigan profundamente, como en el caso de Livia. Y en segundo lugar, una diferenciación, es decir, los modos por los cuales, la conducta y la experiencia siguen desarrollándose y cambiando en respuesta a nuevos contextos. Así, Livia, en sus contactos sociales y también en su relación con Susanna, utilizaba y creía en el "personaje" que solía funcionarle mejor. En terminología de Piaget, en cada encuentro con el mundo, asimilamos la nueva experiencia dentro de nuestros esquemas ya existentes $y$, como parte del mismo proceso, acomodamos esos esquemas a los modos en que la nueva experiencia difiere de las experiencias previas en las cuales los esquemas fueron construidos (Flavell, 1963; Watchel, 1981, 2008). Livia sentía que para beneficio del otro, ella tenía que "aniquilarse", aprendió a interpretar bien el papel de niña estudiosa y después el de una competente profesional. Y como expone Watchel (2008), un niño puede reprimir o negar sus afectos, necesidades, etc., a causa de cómo sean 
respondidos, lo que supone una adaptación defensiva a la percepción de los requerimientos provenientes del otro necesario. Como resultado, uno de los "selfs" de Livia, ya adulta tenía unos sentimientos vacilantes sobre sí misma y su capacidad para poder "experimentar de forma fluida y sin ansiedad la dialéctica entre singularidad y pertenencia". Y no solo eso, además, el niño tiende a volverse excesivamente auto-regulador (Tronick, 1989; Beebe y Lachmann, 1998), en terminología winnicotiana, un "self cuidador", cuando la regulación mutua de los estados internos de un niño es inadecuada. Recordemos cómo Livia se sentía la persona que podría consolar o salvar a su padre y cómo tenía que contener los estados de pánico obsesivo en los que se encontraba la madre.

Podemos observar que las experiencias de interacción de Livia cuando era niña (en terminología de Bolbwy, Fonagy, Ainsworth) no fueron capaces de re-estabilizar sus respuestas emocionales desorganizadas. Para los teóricos del apego, son importantes para el desarrollo las tempranas experiencias de acceso flexible a los sentimientos propios. El control que tiene el niño "seguro" le permite moverse hacia adueñarse de su experiencia interna, y hacia comprenderse a sí mismo y a los otros como seres intencionales cuya conducta está organizada por estados mentales, pensamientos, sentimientos, creencias y deseos (Fonagy et., al 1995; Sroufe, 1990). El contexto familiar de Livia fue un contexto patológicamente falto de límites entre los miembros. Las investigaciones de Karlen LyonsRuth muestran cómo patrones de apego desorganizado pueden predecir procesos disociativos patológicos posteriores. Entendiendo por disociación "poseer una conciencia escasa de la propia subjetividad, es decir, darse poca cuenta de las emociones y pensamientos que se tienen, así como de los hechos emocionalmente relevantes que se han experimentado durante la vida" (Riera, 2011, p. 261). Livia no podía sentir su singularidad, se sentía completamente desprovista de un sentido nuclear de sí misma.

Joan Coderch (2014) expone que una de las razones que impulsan a la mayoría de los pacientes a solicitar tratamiento es la motivación de apego, la búsqueda desesperada del apego seguro que les faltó en la infancia, y estoy de acuerdo con Joan en que "...nuestros pacientes, más allá de las consideraciones razonables que les han llevado a pedir ayuda, esperan encontrar en nosotros esta base de apego seguro" (p. 118). Para desarrollar una adecuada capacidad de mentalización es primordial que las figuras de apego procuren comprender los estados mentales de los hijos y les reflejen esta comprensión. Es decir, los cuidadores que proveen una relación de apego seguro, además de ofrecer a los hijos una disponibilidad atenta, cariñosa y siempre presente, interaccionan con sus hijos de una manera mentalizadora, lo que permite a los niños formar su self (sus múltiples selfs) y adquirir la capacidad de mentalizar. De esta manera los niños adquieren la capacidad de interpretar el comportamiento propio y el de otros a través de la atribución de estados 
mentales (Fonagy et al, 2002). Y probablemente, como expone Guerra (2014), la mentalización sea una de las principales herramientas que nos ayuden a paliar la locura rutinaria en las que nos encontramos en la actualidad. Cuando la figura de apego es capaz de mantener en su mente una representación del niño teniendo sentimientos, deseos e intenciones, permite al pequeño descubrir su propia experiencia interna a través de la representación que la figura de apego construye de la misma. Cuando se da sentido a la experiencia del niño, se le permitirá ir más allá de lo aparente, de lo concreto y se da sentido a su conducta a la luz de los estados mentales (no observables) subyacentes a la misma (Slade, 2008). Todo esto permite que el niño pueda regular su experiencia interna, y desarrollar una representación coherente de sí mismo y de los otros. Parece que en su infancia, Livia no dispuso de esta base de apego seguro, no se dio sentido a su experiencia; recordemos cómo Livia tenía que ser una niña buena, que no diese trabajo, que se portase bien porque su mamá tenía que cuidar a la hermana pequeña y cómo tenía que contener a la madre cuando se encontraba en momentos de pánico obsesivo. Por otro lado, tenía que ser sensata, no llorar para que su padre no se preocupara y tenía que compartir los traumas de su padre. No se permitió a Livia regular su experiencia interna y no pudo desarrollar una representación coherente de sí misma y tampoco de los otros. Como nos expone Susanna, el contexto familiar en que creció Livia, fue un contexto patológicamente falto de límites entre los miembros de su familia y como resultado Livia no tuvo la posibilidad de experimentar de forma fluida y sin ansiedad la dialéctica entre singularidad y pertenencia.

Livia encontró en su relación con Susanna esta base de apego seguro. El diálogo natural y espontáneo entre Livia y Susanna, dos personas que se esfuerzan en entenderse y en encontrarse en la experiencia intersubjetiva (Coderch, J. 2014) favoreció el reconocimiento de múltiples estados del self (Bromberg, 2009; Coderch, 2014; Guerra, 2014). El diálogo que se produjo en terapia entre Susanna y Livia, permitió que se escucharan todas las voces, que se expresaran todos los diversos símismos, estados del self de Livia y, cuando el paciente ve sus distintos estados del self, asumiéndolos como propios, se experimentan a sí mismos con mayor estabilidad y coherencia (Mitchell, 1991, Guerra, 2014). Este diálogo fomenta el reconocimiento mutuo (Benjamín) en la experiencia intersubjetiva $y$, es la conexión emocional entre las personas que dialogan, la directriz fundamental que les guía en este diálogo (Riera, 2011). Paciente y terapeuta conviven íntimamente el uno con el otro en el diálogo analítico e intentan el reconocimiento recíproco de la subjetividad del otro, logrando también un mayor desarrollo y reconocimiento de la propia subjetividad, a la vez que amplían sus horizontes de experiencia (Coderch, 2014). La relación analítica con Susanna, permitió a Livia sentir que era alguien que podía distinguirse de los demás, le permitió experimentar ser sujeto. 
Me gustaría terminar parafraseando a Sandra Buechler:

"Realizar trabajo clínico es una tarea abrumadora. Es un desafío extraordinario tener que concentrarse en tantos niveles a la vez, estar en la vecindad de tanto dolor, soportar la pérdida de cada compañero de tratamiento, y convivir con las limitaciones y los alcances de nuestra influencia. Pero también es un privilegio extraordinario. La formación es tan sólo el comienzo de un esfuerzo que dura toda la vida para convertirnos en los mejores clínicos que podemos llegar a ser. Al igual que el músico, hemos de mantener nuestros instrumentos en plena forma. Pero como clínicos, nuestros instrumentos somos nosotros mismos. Tenemos la audacia y la humildad de intentar usar nuestros propios recursos cognitivos, emocionales e interpersonales para marcar una diferencia en la vida de otros" (2015, p. 441).

\section{REFERENCIAS}

Beebe, B.; Lachmann, F.M.; \& Jaffe, J. (1997). Mother-infant interaction structures and presymbolic self and object representations. Psichoanal. Dial., 7, 133-182

Beebe, B. \& Lachamann, F.M. (1998). Co-constructuing inner and relational processes: Self- and mutual regulation in infant research and adult treatment. Psychoanal. Psychol., 15, 480-516.

Buechler, S. (2015). Marcando la diferencia en las vidas de los pacientes. La experiencia emocional en el ámbito terapéutico. Madrid, Ágora Relacional.

Bromberg, P. (2009). Truth, human relatedness and interpersonal relational process. Int. J. Psychoanal, 90, 347-361.

Coderch, J. (2014). Avances en Psicoanálisis Relacional. Nuevos campos de exploración para el psicoanálisis. Madrid: Ágora Relacional.

Flavel, J.H. (1963). The developmental psychology of Jean Piaget. Princeton, NJ, Van Nostrand.

Fonagy, P., Steele, M., Steele, H., Leigh, T., Kennedy, R., Mattoon, G., \& Target, M. (1995). Attachment, the reflective self, and borderline states: The predictive specificity of the Adult Attachment Interview and pathological emotional development. In S. Goldberg, R. Muir, \& J. Kerr (Eds.). Attachment theory: Social, developmental and clinical perspectives (pp. 233-278). New York: Analytic Press.

Fonagy, G.; Gergely, E., Jurist E. \& Target, M. (2002). Affect Regulation, Mentalization and the Development of the Self. New Yor:, Other Press.

Guerra, L.R. (2014). La insoportable levedad del self. Revista digital de Medicina Psicosomática y Psicoterapia, 4(1). http://www.psicociencias.com/pdf_noticias/La_insoportable_levedad_del_self.pdf 
Mitchel, S. (1991). Contemporary perspectives on self toward and integration. Psychoanatytical dialogues, 1, 121-147.

Riera, R. (2011). La conexión emocional. Cómo se forma nuestra manera espontánea y no voluntaria de reaccional emocionalmente, cómo podemos cambiar esta forma automática de emocionarnos y cuál es el papel de la conexión emocional en estos procesos. Barcelona: Octaedro.

Sroufe, L. A. (1990). An organizational perspective on the self. In D. Cicchetti \& M. Beeghly (Eds.), The self in transition: Infancy to childhood (pp. 281-307). Chicago: University of Chicago Press.

Slade, A. (2008). Working with Parents in Child Psychotherapy: Engaging the Reflective Function. En Frederic N. Busch: Mentalization. Theoretical Considerations, Research Findings and Clinical Implications. The Analytic Press Taylor \& Francis Group, New York, p. 207.

Stern, D.N. (1985). The interpersonal World of the Infant. New York, Basic Books

Stern, S. (2002). The self as a relational structure. A dialogue with Multiple-Self Theory. Psychoanalytic Dialogues, 12 (5), 693-714.

Tronick, E. (1989). Emotions and emotional communication in infants. American Psychologist, 44, 112-119.

Watchel, P.L. (1981). Transference, schema and assimilation: The relevante of Piaget to the psychoanalytic theory of transference. The annual of psychoanalysis, 8, 59-76. New York, International Universities Press.

Watchel, P.L. (2008). Disociación, multiples estados del self e intersubjetividad: psicodinámicas cíclicas y las implicaciones para la teoría relacional. Clínica e investigación relacional, 2(2), 317-342.

Original recibido con fecha: $\quad$ 24/10/2015 Revisado: 20/2/2016 Aceptado: 28/2/2016 